\title{
Non-Linear Forced Vibration of Fully Clamped FGM Skew Plates using Homogenization Technique
}

\section{H. MOULAY ABDELALI ${ }^{1}$, R. BENAMAR ${ }^{2}$}

\author{
1. Université Mohammed V - Ecole Mohammadia D’ingénieurs, Avenue Ibn Sina, B.P.765, Agdal, Rabat, Morocco + \\ h.moulay1983@gmail.com \\ 2. Université Mohammed V - Ecole Mohammadia D’ingénieurs, Avenue Ibn Sina, B.P.765, Agdal, Rabat, Morocco + \\ rhali.benamar@gmail.com
}

\begin{abstract}
The present work concerns the geometrically non-linear forced vibration of fully clamped functionally graded skew plates (FGSP). The theoretical model based on Hamilton's principle and spectral analysis previously applied to obtain the non-linear mode shapes of thin straight structures is used. A homogenization technique is developed to reduce the FGSP problem under consideration to that of an equivalent isotropic homogeneous skew plate. Results are given for the linear and non-linear fundamental frequency of fully clamped FGSP, considering different parameters, such as the skew angle, the excitation force level. The results show a non linearity of the hardening type with a shift to the right of the bent non linear frequency response function, in the neighbourhood of the fundamental mode.
\end{abstract}

Keywords : linear vibrations, FGM skew plate, Homogenization technique, Forced vibration.

\section{Introduction}

Classical composite materials now currently used in many industrial fields are sometimes not very appropriate for use under high-temperature conditions. The functionally graded materials possess the best qualities of their constituents, i.e. the strength and toughness of the metal and the excellent heat resistance of the ceramic. Since these materials are non homogeneous, they offer a possible decrease in the inplane and through-the thickness transverse stresses, in addition to the improved thermal behaviour owing to low thermal conductivity of the ceramic component. The FGM are actually used in various fields such as medicine, civil engineering, aeronautic and aerospace.
The dynamics of rectangular FG plates has been investigated by many researchers in the linear regime but nonlinear vibrations of FGSP have been less investigated. Fazzolari and Carrera [1] developed a fully coupled thermo-elastic formulation to deal with the free vibration analysis of anisotropic composite and isotropic/sandwich FG plates. Zhang and Zhou [2] presented a theoretical analysis of FG thin plates, based on the physical neutral surface. The physical neutral surface of a FG plate was determined by the classical thin plate theory. Geometrically non-linear free and forced vibration of fully clamped FGSP is examined here using a homogenization technique, in order to investigate the effect of non-linearity on the amplitude dependent non-linear resonance frequencies and associated nonlinear fundamental mode shape. The non-linear resonance frequency ratio $\omega \mathrm{nl} / \omega \mathrm{l}$ is determined for various values of the plate skew angle and a wide range of vibration amplitudes. Large deflection responses of functionally graded rectangular plates under transverse loads is investigated by using a semi-analytical approach and compared with previous results.

\section{Non-Linear Forced Vibration of Fully Clamped FGSP}

Consider the FGSP shown in Fig. 1 with a skew angle $\theta$ and a plate thickness $\mathrm{H}$. Metal and ceramics are the two isotropic materials constituting the skew plate, with a composition varying from the plate top surface $(z=H / 2)$, which is ceramic rich, to the bottom surface $(z=-H / 2)$, which is metal rich. The plate thickness is considered to be sufficiently small so as to avoid the effects of shear 
deformation. The skew plate has the following characteristics: a, b, S: length, width and area of the plate; $x-y$ : plate co-ordinates in the length and the width directions; $\xi-\eta$ : Skew plate co-ordinates.

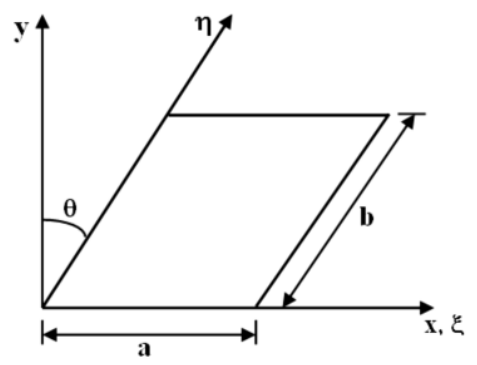

Fig.1. FGSP in $x-y$ and $\xi-\eta$ co-ordinate system

The elastic material properties vary through the plate thickness according to the volume fractions of the constituents. The variation of material properties is described by a Power-law distribution expressed as:

$$
\begin{aligned}
& \mathrm{P}(\mathrm{z})=\left(\mathrm{P}_{\mathrm{c}}-\mathrm{P}_{\mathrm{m}}\right) \mathrm{V}_{\mathrm{c}}(\mathrm{z})+\mathrm{P}_{\mathrm{m}} \\
& \mathrm{V}_{\mathrm{c}}(\mathrm{z})=\left(\frac{\mathrm{z}}{\mathrm{h}}+\frac{1}{2}\right)^{\mathrm{n}}(0 \leq \mathrm{n} \leq \infty)
\end{aligned}
$$

Where $\mathrm{P}$ represent the material property, $\mathrm{Pm}$ and $\mathrm{Pc}$ represent the properties of the metal and ceramic, respectively; $\mathrm{Vc}$ is the ceramic volume fraction and $\mathrm{n}$ is the volume fraction exponent. The power-law is used as a simple mixture rule to express the variation of the effective properties of the ceramic-metal in the thickness direction of the FGSP. The temperature is assumed to be equal to $300\left({ }^{\circ} \mathrm{K}\right)$ and the materials used are Zirconia $(\mathrm{ZrO} 2)$ on the top and aluminum on the bottom.

\section{Results and Discussion}

The aim of this section is to apply the theoretical model presented in ref [3] and [4] to analyze the geometrical non-linear free and forced dynamic response of fully clamped FGSP. The effects of non-linearity on the nonlinear resonance frequencies and non linear fundamental mode shape at large vibration amplitudes are investigated. Convergence studies were carried out, and the results are compared with those available from the literature through few examples of FGSP. Calculation was made by using 18 plate functions. the strain energy due to bending $\mathrm{Vb}$, the membrane strain energy $\mathrm{Vm}$, the coupling strain energy $\mathrm{Vc}$ and the kinetic energy $\mathrm{T}$ are given in skew coordinate wich are related to the rectangular co-ordinate $(\xi, \eta)$ by: $\xi=x-y \tan \theta ; \eta=y / \cos \theta$. In order to simplify the expressions and reduce the problem to that of a homogeneous skew plate, the change of variable $\mathrm{z} 1=\mathrm{Z}-\mathrm{c}$ is made, With $\mathrm{c}=\frac{\mathrm{B}_{11}}{\mathrm{~A}_{11}}$. $\mathrm{A}$ and $\mathrm{B}$ are the extensional and coupling stiffness coefficients. Consequently, the coupling term $\mathrm{Vc}$ vanishes.Table 1 shows the linear results of a fully clamped FGSP for different values of volume fraction $\mathrm{N}$ and a skew angle $\theta=0^{\circ}$ and an aspect ratio $\alpha=1$. It can be noticed that the adimensional frequencies decreases with values of volume fraction $\mathrm{N}$.

Table 1: Adimensional linear frequencies of fully clamped FGM square plate for different values of volume fraction $\mathrm{N}$

\begin{tabular}{|c|c|}
\hline $\mathbf{N}$ & $\boldsymbol{\omega}^{*}$ linear \\
\hline 0 & 64,20 \\
\hline 0,5 & 57,71 \\
\hline 1 & 52,78 \\
\hline 2 & 46,10 \\
\hline 5 & 35,09 \\
\hline 10 & 26,94 \\
\hline
\end{tabular}

Table 2 shows the non dimensional nonlinear frequency results for fully clamped FGSP with different values of volume fraction $\mathrm{n}$ and different non dimensional nonlinear maximum displacement $\mathrm{W}^{*} \max$. It can be noticed that the non dimensional nonlinear frequency ratios increase with the volume fraction $\mathrm{n}$ and with the non dimensional non-linear maximum displacement $\mathrm{W}^{*} \max$, which corresponds to a non linearity of the hardening type.

Table 2: Non dimensional nonlinear frequency ratios of fully clamped FGM square plate for different values of volume fraction $\mathrm{n}$ and for different values of the non dimensional nonlinear maximum displacement $\mathrm{W}^{*} \max$

\begin{tabular}{|c|c|c|c|c|c|c|}
\hline & \multicolumn{6}{|c|}{$\boldsymbol{\omega}^{*} \mathbf{n} \mathbf{\omega} \mathbf{* 1}$} \\
\hline $\mathbf{W}$ max & $\mathbf{N = \mathbf { 0 }}$ & $\mathbf{N = 0 , 5}$ & $\mathbf{N}=\mathbf{1}$ & $\mathbf{N}=\mathbf{2}$ & $\mathbf{N}=\mathbf{5}$ & $\mathbf{N = 1 0}$ \\
\hline 0 & 1 & 1 & 1 & 1 & 1 & 1 \\
\hline 0,2 & 1,0051 & 1,0063 & 1,0075 & 1,0098 & 1,0169 & 1,0285 \\
\hline 0,4 & 1,0201 & 1,0249 & 1,0296 & 1,0387 & 1,0659 & 1,1095 \\
\hline 0,6 & 1,0448 & 1,0551 & 1,0655 & 1,0851 & 1,1430 & 1,2328 \\
\hline 0,8 & 1,0783 & 1,0961 & 1,1139 & 1,1470 & 1,2429 & 1,3871 \\
\hline 1 & 1,1199 & 1,1466 & 1,1731 & 1,2219 & 1,3605 & 1,5633 \\
\hline
\end{tabular}




\begin{tabular}{|c|c|c|c|c|c|c|}
\hline & \multicolumn{6}{|c|}{$\omega^{*} \mathrm{nl} / \omega^{*} \mathbf{I}$} \\
\hline $\mathrm{W}^{*} \max$ & $\mathrm{N}=0$ & $\mathrm{~N}=\mathbf{0 , 5}$ & $N=1$ & $\mathrm{~N}=2$ & $N=5$ & $\mathrm{~N}=10$ \\
\hline 1,2 & 1,1688 & 1,2054 & 1,2416 & 1,3076 & 1,4918 & 1,7547 \\
\hline 1,4 & 1,2241 & 1,2715 & 1,3180 & 1,4022 & 1,6334 & 1,9570 \\
\hline 1,6 & 1,2849 & 1,3436 & 1,4010 & 1,5040 & 1,7829 & 2,1671 \\
\hline
\end{tabular}

Fig.2 shows the non dimensional non-linear maximum displacement $\mathrm{W}^{*} \max$ of $\mathrm{FG}$ square plate $\left(\mathrm{N}=1, \theta=0^{\circ}\right.$ and $\alpha=1)$ versus the nonlinear frequency ratio. The comparison made between the results obtained in reference [3] and the present ones shows a good agreement.
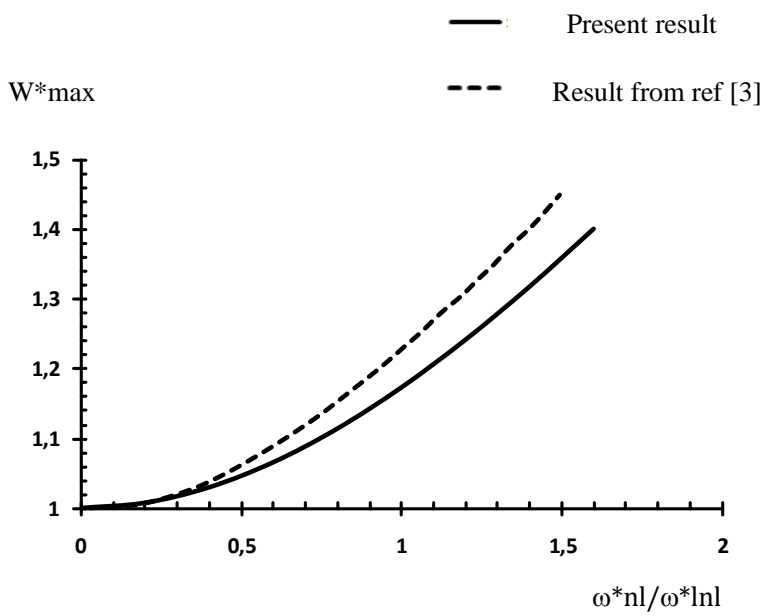

Fig.2. Non dimensional non-linear maximum displacement $\mathrm{W}^{*} \max$ of FG square plate $\left(\mathrm{N}=1, \theta=0^{\circ}\right.$ and $\left.\alpha=1\right)$ versus the nonlinear frequency ratio

Fig.3 shows the effect of the volume fraction exponent on the vibration of a fully clamped FGM skew plate $\left(\theta=30^{\circ}\right)$ subjected to a harmonic distributed force $\mathrm{f}_{1} * \mathrm{~d}=100$. It can be seen that the nonlinearity increase with the volume fraction exponent.
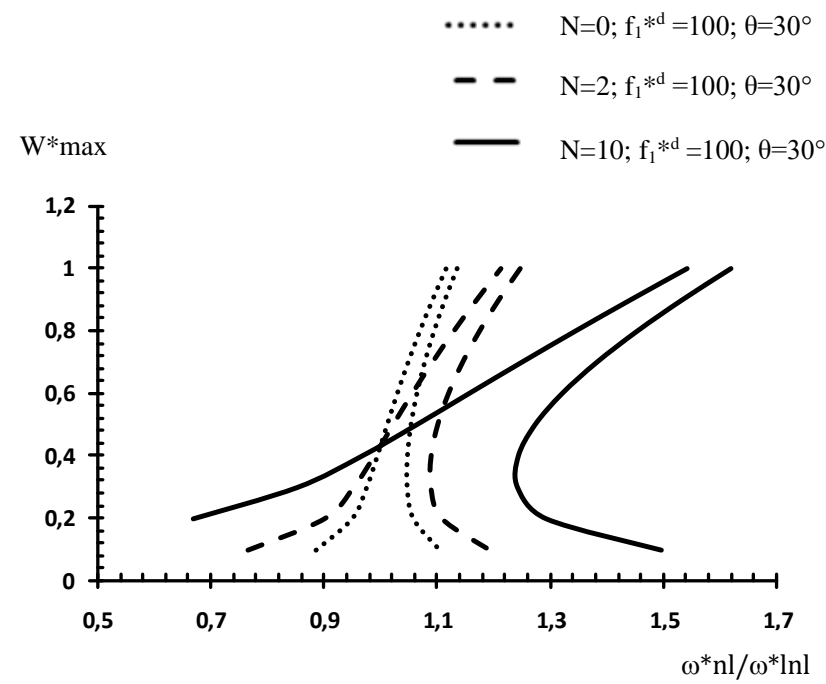

Fig.3. Effect of the volume fraction exponent on the forced vibration of a fully clamped FGM skew plate $\left(\theta=30^{\circ}\right)$ subjected to harmonic distributed force $\mathrm{f}_{1} * \mathrm{~d}=100$

\section{Conclusion}

The theoretical model established and applied previously to beams, plates and shells [4], has been successfully applied to calculate the first non-linear mode shape of fully clamped FGSP. A homogenization technique was applied for geometrical non-linear, steady state, periodic forced vibration of FGM skew plates for various values of the volume fraction exponent and the skew angle. The present formulation has been verified with the results available in the literature. The effect of the volume fraction index $\mathrm{n}$, the skew angle $\theta$ has been discussed. Good results were found using a single and multimode approach to determine the amplitude frequency dependence in the centre of the plate by varying the skew angle and the volume fraction exponent. It can be seen that increasing the skew angle reduces the effect of nonlinearity and increasing the volume fraction exponent decreases the nonlinearity. Good agreement between the present results and those found in the literature has been achieved. The present study reveals a hardening type of vibration.

\section{References}

[1] F. A. Fazzolari, E. Carrera, Coupled thermoelastic effect in free vibration analysis of anisotropic multilayered plates and FGM plates by using a variable-kinematics Ritz formulation, European Journal of Mechanics A/Solids, Vol. 44 (2014), p. 157-174

[2] D. Zhang, Y. Zhou, A theoretical analysis of FGM thin plates based on physical neutral surface, Computational Materials Science, Vol. 44 (2008), p. 716-720

[3] A. Boukhzer, K. EL Bikri and R. Benamar, Homogenization technique for non-linear free vibrations analysis of FGM rectangular plates, Advanced Materials Research Vols. 971-973 (2014) p. 516-533.

[4] R. Benamar, M.M. K. Bennouna and R. G. White, The effects of large vibration amplitudes on the fundamental mode shape of thin elastic structures, Part II fully clamped rectangular isotropic plates, Journal of Sound and Vibration Vol. 164 (1993), p.295-316 